\title{
ARTICLE
}

Epidemiology

\section{The clinical and biological significance of HER2 over-expression in breast ductal carcinoma in situ: a large study from a single institution}

\author{
Islam M. Miligy ${ }^{1,2}$, Michael S. Toss ${ }^{1,3}$, Kylie L. Gorringe ${ }^{4,5}$, Andrew H. S. Lee ${ }^{1}$, Ian O. Ellis ${ }^{1}$, Andrew R. Green ${ }^{1}$ and Emad A. Rakha ${ }^{1,2}$
}

BACKGROUND: Previous studies have reported up to 50\% of ductal carcinoma in situ (DCIS), is HER2 positive, but the frequency of HER2-positive invasive breast cancer (IBC) is lower. The aim of this study is to characterise HER2 status in DCIS and assess its prognostic value.

METHODS: HER2 status was evaluated in a large series of DCIS $(n=868)$, including pure DCIS and DCIS associated with IBC, prepared as tissue microarrays (TMAs). HER2 status was assessed using immunohistochemistry (IHC) and chromogenic in situ hybridisation (CISH).

RESULTS: In pure DCIS, HER2 protein was over-expressed in 9\% of DCIS (3+), whereas 15\% were HER2 equivocal (2+). Using CISH, the final HER2 status was positive in $20 \%$. In mixed DCIS, HER2 amplification of the DCIS component was detected in $15 \%$ with amplification in the invasive component of only $12 \%$. HER2-positive DCIS was associated with features of aggressiveness $(p<0.0001)$ and more frequent local recurrence $(p=0.03)$. On multivariate analysis, combined HER $2+/$ Ki67 + profile was an independent predictor of local recurrence $(p=0.006)$.

CONCLUSIONS: The frequency of HER2 positivity in DCIS is comparable to IBC- and HER2-positive DCIS is associated with features of poor prognosis. The majority of HER2 over-expression in DCIS is driven by gene amplification.

British Journal of Cancer (2019) 120:1075-1082; https://doi.org/10.1038/s41416-019-0436-3

\section{BACKGROUND}

Ductal carcinoma in situ (DCIS) of the breast was less common before the 1980s; however, with the introduction of mammographic screening, the incidence has increased dramatically and now comprise approximately $20 \%$ of breast cancers. ${ }^{1}$ Over the last decade, breast carcinoma in situ incidence rates have increased by almost half (46\%) in the UK (http://www.cancerresearchuk.org/ health-professional/cancer-statistics). The mortality from DCIS is very low, with a maximum of $3 \%$ at 20 years of follow-up and the survival after surgery is excellent. ${ }^{2}$

Following a diagnosis of DCIS, women are at elevated risk for disease progression, ${ }^{3}$ but not all DCIS cases progress to invasive carcinoma if untreated with an estimated risk in the range of $25-50 \%$. ${ }^{4}$ Therefore, the main aim of DCIS management is to prevent progression and local recurrence, particularly invasive recurrence. $^{5}$

Clinicians are unable to precisely predict the risk of local recurrence or progression to invasive cancer in patients with DCIS following their treatment, a major concern that needs to be addressed. Treatment is by mastectomy, or lumpectomy with or without adjuvant radiotherapy. ${ }^{6}$ Factors such as young patient age, large tumour size, ${ }^{7}$ positive margins, comedo necrosis and high-nuclear grade ${ }^{8}$ are associated with a higher risk of recurrence with some emerging data suggesting that ERBB2-amplified (HER2) DCIS could present a higher risk of recurrence. ${ }^{9}$ The Van Nuys Prognostic Index is a popular risk assessment tool combining patient age, lesion size, nuclear grade and margin status for treatment decisions. ${ }^{10}$

HER2 is an established negative prognostic factor in invasive breast cancer. ${ }^{11}$ The prognostic significance of HER2 status in DCIS is, however, less clear. ${ }^{1,12}$ The relationship of HER2 to risk of recurrence and its role in the progression from in situ to invasive cancer have been debated. ${ }^{9}$ HER2 over-expression is reported to be more frequent in DCIS than in invasive cancer. ${ }^{13}$ This may seem contradictory, as HER2 is proposed to play a role in tumour progression. Some studies report an even higher proportion of HER2 positivity in microinvasive cancer ${ }^{14}$ and, in preoperative tumour biopsies displaying DCIS, HER2 over-expression has been related to increased incidence of invasive carcinoma in the surgical specimen. ${ }^{15}$ Furthermore, HER2 positivity is associated with high-histopathological grade both in invasive cancer and in DCIS. ${ }^{16}$

Immunohistochemical staining (IHC) can be used as a surrogate marker of gene-expression profiling in invasive breast cancer,

\footnotetext{
${ }^{1}$ Nottingham Breast Cancer Research Centre, Division of Cancer and Stem Cells, School of Medicine, Nottingham City Hospital, The University of Nottingham, Nottingham, UK;

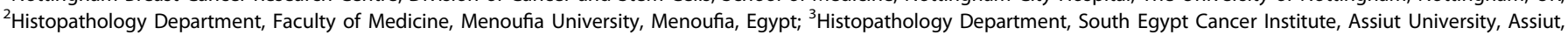

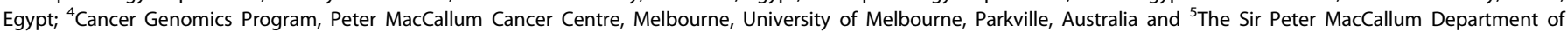
Oncology, University of Melbourne, Parkville, Australia

Correspondence: Emad A. Rakha (emad.rakha@nottingham.ac.uk, Emad.rakha@nuh.nhs.uk)
}

Received: 8 September 2018 Revised: 4 March 2019 Accepted: 7 March 2019

Published online: 8 May 2019 
utilising ER, PR, HER2 and Ki-67 protein expression to split patients into four different molecular phenotypes. ${ }^{17}$ Similar subtypes have been found in DCIS, but the prognostic significance of grouping DCIS into these subtypes is not yet clear. Some studies have suggested the most appropriate treatment could be identified by evaluating individual expression of genes or receptors in DCIS. ${ }^{18}$

IHC staining is the predominant method of determining HER2 status in breast cancer specimens due to its relative ease to perform with rapid turnaround time and lower cost. ${ }^{19}$ Although commercially available antibodies have shown wide variation in sensitivity and specificity for formalin-fixed paraffin-embedded (FFPE) tissue samples, in which tissue fixation and pre-treatment have a substantial effect on staining, ${ }^{20,21}$ a significant improvement of the diagnostic standards in clinical histopathology with quality control measures were able to improve diagnostic performance in clinical practice. ${ }^{22}$

HER2 gene amplification is primarily detected by in situ hybridisation and uses fluorescence (FISH) to detect the signals. This method is expensive and requires expertise with fluorescent microscopy using appropriate filters. ${ }^{23}$ Chromogenic in situ hybridisation (CISH) offers an alternative method, and whilst it utilises in situ hybridisation technology of FISH, it takes advantage of a chromogenic signal detection that is assessed using light microscopy and therefore costs much less than FISH. CISH is able to detect HER2 gene amplification and to minimise, if not eliminate, the borderline category with $\mathrm{IHC}^{24}$ The performance of CISH in HER2 testing has rarely been tested in series, so we aimed to characterise HER2 status using IHC and CISH in DCIS and assess its prognostic value in a large and well annotated cohort.

\section{METHODS}

Study cohort and tissue microarray

This retrospective study was conducted on a consecutive series of primary pure DCIS cases diagnosed, and treated, between 1990 and 2012 at Nottingham City Hospital, Nottingham, UK. Exclusion of referral, miscoded and recurrent cases resulted in 776 cases of pure primary DCIS with available (FFPE tumour blocks for TMA construction. During the same time-period, a series of 239 cases diagnosed as synchronous DCIS and invasive tumours (mixed DCIS and invasive breast cancer (IBC)) was also collected for comparison. Patients' demographic information, histopathological parameters, surgical management, radiotherapy and patient outcome data were collated. Tumour size was the sum of the primary excision and the re-excision tumour size $(\mathrm{mm})$. Tumour grade was classified according to the three-tier nuclear grading system; low, intermediate and high-nuclear grades. ${ }^{25}$ Local recurrence free survival was calculated based on the time (in months) from the date of primary surgical treatment to the time of ipsilateral local recurrence. Patients who developed contralateral disease following DCIS diagnosis were censored at the time of diagnosis of the contralateral cancer.

The median follow-up period in the pure DCIS cohort treated with breast conserving surgery was 118 months (range: 2-240 months), during which 75 patients (12\%) developed ipsilateral local recurrence, including invasive carcinoma in 48 (62\%). Of the 308 patients treated with breast conserving surgery alone, there were 67 recurrences (22\%), of which 38 (57\%) cases were invasive recurrences. Amongst the 93 cases treated with breast conserving surgery and radiotherapy, there were 8 recurrences (9\%) (2 invasive and 6 DCIS).

Tissue microarrays (TMAs) were prepared from representative DCIS lesions of the pure cases and from DCIS and invasive tumours from the mixed cases as previously described. ${ }^{26}$ The TMA was constructed using 3D Histech Grand Master ${ }^{\oplus}$, Budapest,
Hungary, whereby representative cores of 1 and $0.6 \mathrm{~mm}$ from appropriate viable tumour area were taken from DCIS and invasive tumour samples, respectively, avoiding scanty, single focus, necrotic tumour zones and thinner tumour blocks than $4 \mathrm{~mm}$. Each case was represented by a single-core biopsy; however, cases with more than one histologic tumour type were represented by more than one core.

Immunohistochemistry and chromogenic in situ hybridisation The expression of ER, PR, HER2 and Ki67 was evaluated using IHC on the TMA sections. Positive and negative controls were included in every assay (Supplementary material). MIB1 antibody was used for evaluation of Ki67 expression. Nuclear staining was scored for ER, PR and Ki67 and membrane staining for HER2. HER2 status was assessed using the HercepTest scoring method $^{27}$ where 0 (no membrane staining or incomplete staining of $<10 \%$ of cells), $1+$ (weak or moderate incomplete membrane staining of $>10 \%$ cells), $2+$ (strong, complete membrane staining in $\leq 10 \%$ of tumour cells or weak/moderate complete membrane staining in $\geq 10 \%$ of tumour cells) or $3+$ (strong, complete membrane staining in $>10 \%$ of tumour cells). HER2 status was considered negative if the immunohistochemical score was 0 or $1+$, equivocal if the score was $2+$ and positive if the score was $3+$.

In equivocal cases, HER2 gene amplification was determined by $\mathrm{CISH}$. This was performed using the ZytoDot 2C SPEC ERBB2/CEN 17 Probe Kit, Germany. Interpretation of $\mathrm{CISH}$ was performed using a Nikon microscope equipped with a $\times 63$ objective lens. At least 20 malignant, nonoverlapping cell nuclei were scored to assess HER2 gene copy number. Chromosome 17 (represented by the red signals) was used as an internal control particularly in the low HER2 gene copy number cases as per the recommended protocol. HER2 gene amplification was defined as six or more signals per nucleus or when clusters (clumps of aggregated green signals) were identified in the cell nuclei in more than $50 \%$ of tumour cells. ${ }^{28}$ If the average copy number was $\geq 4$ to $<6$ per nucleus (equivocal), another 20 tumour cells were enumerated, and the final average copy number of the case was calculated from the total of 40 cells. In cases with multiple cores $(n=26)$, HER2 score was initially obtained by IHC followed by confirmation with $\mathrm{CISH}$ to reach the final status of the case (Supplementary Table 1).

For ER and PR, a 1\% cut-off value was used to dichotomise cases into positive and negative. ${ }^{29}$ For Ki67 the cut-off for differentiating low- and high-proliferative groups was $14 \% .^{30}$

\section{Statistical analysis}

Statistical analyses were performed using SPSS v24 (Chicago, IL, USA) for windows. Association between final HER2 status and clinicopathological parameters using categorised data in the pure DCIS cohort was evaluated using chi-squared test. Survival rates were determined using the Kaplan-Meier method and compared by the log-rank test. Multivariate survival analyses using Cox proportional hazard regression model was used to evaluate the associations between independent variables and local recurrence. Multivariable hazards ratio and their corresponding 95\% confidence intervals $(\mathrm{Cl})$ were initially performed for each marker alone and then for the combination of biomarkers. All tests were two-tailed and a $p$ value of less than 0.05 was considered as statistically significant.

\section{RESULTS}

A total of 651 cases of pure DCIS patients (Supplementary Table 2) and 217 cases mixed with invasive carcinoma were suitable for analysis. When pure DCIS was compared to DCIS mixed with invasive disease, mixed DCIS was of higher nuclear grade with comedo necrosis $(p<0.0001)$. 
Assessment of HER2 status

In the pure DCIS group, $20 \%$ of cases were HER2 positive. Immunohistochemistry for HER2 was scored as $3+$ in $9 \%, 2+$ in $15 \%$ and 0 or $1+$ in $76 \%$ (Table 1). All $3+$ cases showed HER2 amplification with $\mathrm{CISH}$. Totally, $73 \%$ of $2+$ cases showed HER2 amplification (4 cases in which CISH could not be assessed due to technical issues and were excluded) (Fig. 1). CISH confirmed highcopy number of HER2 gene in all IHC 3+ cases. Of the 493 cases with $0 / 1+$ score using IHC, only one case showed HER2 amplification. When $\mathrm{IHC}$ results were assessed with the exclusion of equivocal $(2+)$ tumours, agreement between IHC and CISH in 0 , $1+$ and $3+$ cases was $99.7 \%(\kappa$-coefficient $=0.997)$ (Table 1 and Supplementary Table 3). Interestingly, when the overall rate of positivity was analysed over the period of the study, the frequency of positivity reduced from $30 \%$ in the 1990 s to $20 \%$ in 2012 (Fig. 2).

Twenty-six cases had multiple cores across the TMAs, of which 15 were concordant for IHC and CISH ( 13 for $\mathrm{IHC} 0 / 1+$ and 2 for IHC $2+$ ). Of the 11 cases with discordant IHC, which included all possible pairwise combinations of $0 / 1+, 2+$ and $3+$, the $\mathrm{CISH}$ results were consistent across the cores, indicating a greater reliability of $\mathrm{CISH}$ over $\mathrm{IHC}$ in detecting true amplification particularly in heterogeneous cases as well as in limited samples such as core biopsies.

In mixed DCIS and invasive carcinoma cases, the DCIS component was HER2 positive in $15 \%$ and the invasive component in $12 \% \quad(p=0.051$, Table 2 and Supplementary Tables 4-7). All eight discordant cases had extensive DCIS; six of them were of high grade and ER positive.

Table 1. HER2 status in pure DCIS cases using IHC and CISH

\begin{tabular}{lllll}
\hline CISH & \multicolumn{3}{l}{ HER2 IHC in pure DCIS } & \\
\cline { 2 - 5 } & $\begin{array}{l}0 / 1+ \\
(n=493)(\%)\end{array}$ & $\begin{array}{l}2+ \\
(n=98)(\%)\end{array}$ & $\begin{array}{l}3+ \\
(n=56)(\%)\end{array}$ & $\begin{array}{l}\text { Total } \\
(n=647)(\%)\end{array}$ \\
\hline Amplified & $1(0.2)$ & $72(73.5)$ & $56(100.0)$ & $129(20.0)$ \\
Non-amplified & $492(99.8)$ & $26(26.5)$ & $0(0.0)$ & $518(80.0)$ \\
\hline
\end{tabular}

DCIS ductal carcinoma in situ, IHC immunohistochemistry, CISH chromogenic in situ hybridisation
Correlation with clinicopathological parameters and molecular biomarkers

In pure DCIS, HER2 positivity was associated with larger tumour size, high-nuclear grade, comedo type DCIS, negative hormone receptor status, high-Ki67 proliferation index and abnormal expression of $\mathrm{p} 53$ (all $p<0.0001$ ). HER2-positive cases were more frequent in patients treated by mastectomy $(p=0.001)$ (Table 3$)$.

Associations with outcome

Nuclear grade, comedo necrosis and margin width were individually associated with the development of all ipsilateral recurrences ( $p=0.023,0.013$ and 0.029 , respectively). When recurrences were stratified into in situ and invasive recurrences, HER2 was associated with the development of DCIS local recurrence. Patients with HER2+/Ki67-high DCIS had a high likelihood of developing invasive local recurrence $(p=0.004)$. On multivariable analysis, patients with HER2+/Ki67-high DCIS had a hazard ratio of 2.5 (95\% Cl: 1.7-55.0, $p=0.006)$, compared to women with other profiles (Table 4).

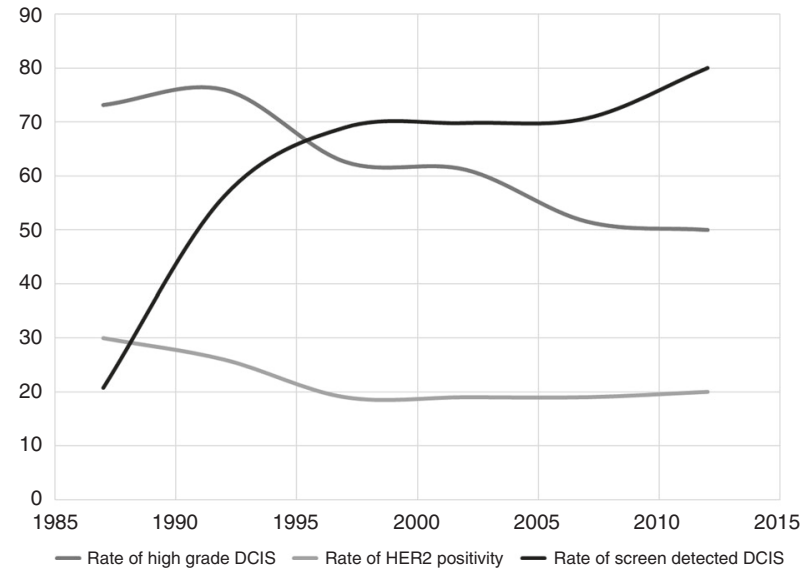

Fig. 2 The rate of HER2 positivity, grade and screen detected DCIS change over time. The graph shows that the rate of high grade DCIS together with HER2 positivity is declining over years in contrast to the rate of screen detected DCIS a

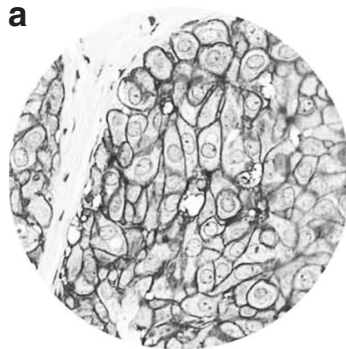

b

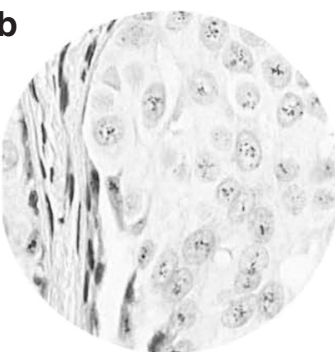

c

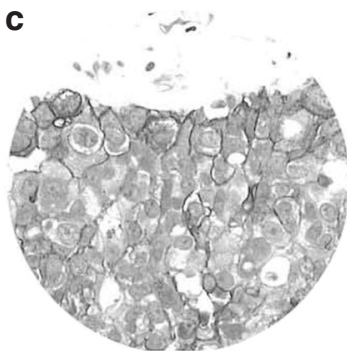

d

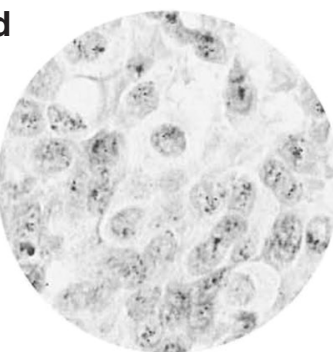

e

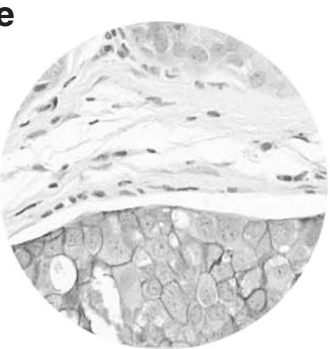

f

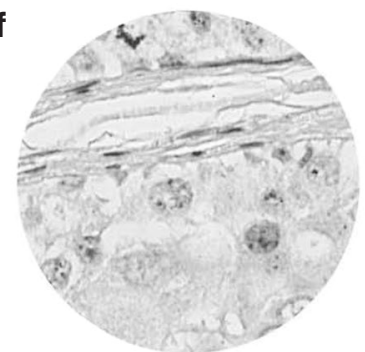

g

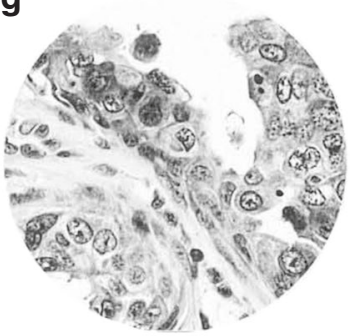

h

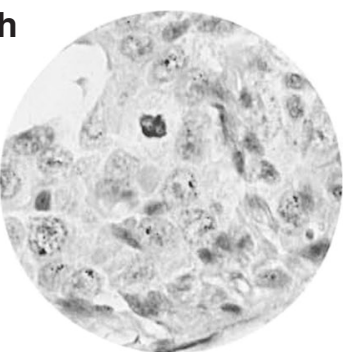

Fig. 1 DCIS showing HER2 3+ (a) confirmed by CISH (b), HER2 2+ (c) showing gene amplification (d), HER2 2+ (e) without amplified gene (f) and HER2 $1+(\mathbf{g})$ showing no gene amplification (h) 
Table 2. HER2 status in DCIS cases mixed with invasion

(A) HER2 status in DCIS cases mixed with invasion using IHC and CISH

\begin{tabular}{|c|c|c|c|c|c|c|c|c|}
\hline \multirow{2}{*}{$\mathrm{CISH}$} & \multicolumn{4}{|c|}{ HER2 IHC DCIS component } & \multicolumn{4}{|c|}{ HER2 IHC invasive component } \\
\hline & $\begin{array}{l}1+(n=185) \\
(\%)\end{array}$ & $\begin{array}{l}2+(n=10) \\
(\%)\end{array}$ & $\begin{array}{l}3+(n=22) \\
(\%)\end{array}$ & $\begin{array}{l}\text { Total }(n=217) \\
(\%)\end{array}$ & $\begin{array}{l}1+(n=192) \\
(\%)\end{array}$ & $\begin{array}{l}2+(n=2) \\
(\%)\end{array}$ & $\begin{array}{l}3+(n=23) \\
(\%)\end{array}$ & $\begin{array}{l}\text { Total }(n=217) \\
(\%)\end{array}$ \\
\hline Amplified & $5(2.7)$ & $5(50.0)$ & $22(100.0)$ & $32(14.7)$ & $1(0.5)$ & $1(50.0)$ & $23(100.0)$ & $25(11.5)$ \\
\hline Non-amplified & $180(97.3)$ & $5(50.0)$ & $0(0.0)$ & $185(85.3)$ & 191 (99.5) & $1(50.0)$ & $0(0.0)$ & 192 (88.5) \\
\hline
\end{tabular}

(B) Final HER2 status of DCIS and invasive component of DCIS cases mixed with invasion

\begin{tabular}{llll}
\hline & Invasive HER2 & & Total no. (\%) \\
\cline { 2 - 3 } DCIS HER2 & Positive & Negative & 184 \\
\hline Negative & 1 & 8 & $185(85.3)$ \\
Positive & 24 & $192(88.5)$ & $32(14.7)$ \\
Total & $25(11.5)$ & 217 \\
\hline
\end{tabular}

DCIS ductal carcinoma in situ, IHC immunohistochemistry, CISH chromogenic in situ hybridisation

\section{DISCUSSION}

HER2 status can be detected by analysing the number of gene copies or measuring the amount of protein expression. The most widely used methods at present are IHC, FISH and CISH due to their propensity in evaluating HER2 in FFPE tissues in invasive carcinoma. ${ }^{31}$ Most HER2 studies have been performed by IHC, which is widely accessible, easy to perform at a reasonable cost and is appropriate for initial HER2 assessment. ${ }^{32}$ However, in cases with an $\mathrm{IHC}$ score of $2+$, the inter-observer agreement is poor, and the predictive value is unsatisfactory for clinical use. This led to the recommendation of additional testing measuring HER2 gene copy number status using an in situ hybridisation technique such as FISH or CISH to avoid inaccurate prognostication and inappropriate treatment. 32,33

Although FISH is a fairly objective and quantitative procedure in detecting HER2 gene amplification, it has many drawbacks, including its cost, the essential need for a fluorescence microscope, its temporary signal which requires a special camera and is technically challenging with TMA preparations. ${ }^{34} \mathrm{CISH}$ is an alternative in situ hybridisation method to analyse gene amplification. ${ }^{35}$ It requires an ordinary bright field microscope and the method is less cumbersome and more economical with permanent signal intensity. Pathologists are able to correlate findings with the underlying tumour histo-morphology. Furthermore, several studies have demonstrated good correlation between $\mathrm{CISH}$ and FISH results. ${ }^{36,37} \mathrm{CISH}$ is either performed as dual colour or single colour with an advantage of the latter over the former in being easy to count signals especially in amplified cases. $^{38}$

To the best of our knowledge, this is the first study to assess HER2 in DCIS using in situ hybridisation. In this study, similar to a previous study, but in invasive tumour, ${ }^{31}$ the concordance between $3+\mathrm{IHC}$ and $\mathrm{CISH}$-amplified cases was $100 \%$, denoting all HER2 over-expressing cases as having HER2 gene amplification. In contrast, the proportion of $2+\mathrm{IHC}$ that were HER2 amplified, as detected by $\mathrm{CISH}$, was $73.5 \%$, which is lower than previously reported $(93 \%){ }^{39}$ The $26.5 \%$ (26/98) IHC-equivocal/HER2 nonamplified tumours in this study, all of which had $2+\mathrm{IHC}$ scores, is higher than the result obtained by other studies on invasive tumours. ${ }^{40-42}$ The concordance between $0 / 1+$ IHC and HER2 nonamplified tumours was $99.7 \%$. A similar finding was reported by Zhao and colleagues and they considered that there is a small undetermined percentage of amplified HER2 without protein overexpression. ${ }^{40}$ The same study and other FISH studies on invasive carcinoma also reported rare $3+\mathrm{IHC}$ tumours that were nonamplified, ${ }^{40-43}$ however, we did not detect any such cases.

The low-amplified HER2 category ( $\geq 4$ to $<6 \mathrm{CISH}$ signals) was the most difficult to interpret, requiring an accurate enumeration of gene copy and addition of more tumour cells to assess the final status. This step was particularly important because these cases could either resolve as amplified (4/7) or non-amplified (3/7). Signal clustering, more probably a result of intra-chromosomal amplification of homogeneously staining regions, was immediately evident in highly amplified cases and was easily evaluated.

We observed a lower HER2 expression in DCIS compared to the previously published literature. ${ }^{1,15}$ This might be related to the increasing detection of low- and intermediate-grade DCIS by screening mammography. In support of this the HER2 positivity was higher $(30 \%)$ in the earlier period of the study and declined over time to $20 \%$ (Fig. 2).

The question of whether or not TMA cores are representative of whole tumours is frequently raised, as some tumours are heterogeneous and so a small sample of tissue may not always display the same biological characteristics as a larger section. ${ }^{34}$ To increase the credibility of the TMA technique while reducing the probability of error associated with the difficulty of obtaining a representative sample, some studies use multiple cores from the same donor tissues as it has been reported that only a few (up to four) cores are required to achieve a $100 \%$ agreement. ${ }^{44}$ However, even when as many as ten tissue cores are taken from a tumour, some disagreement may be noted. ${ }^{45,46}$ In the current study, $1 \mathrm{~mm}$ diameter cores were used to construct the TMA of the pure DCIS tumours, increasing the probability of accurate sampling by taking large TMA cores. In cases with multiple cores, the initial HER2 status was assessed at the protein level followed by confirmation with in situ hybridisation. The high agreement rate between IHC and $\mathrm{CISH}$ results suggests that it is possible to apply TMAs to DCIS research, with the caveat that, for HER2, the CISH results may be more reliable, countering the discrepancy that can be observed in IHC for either technical or biological reasons.

Our subsequent goal in this study was to assess the role of HER2 in progression of DCIS. HER2-positive DCIS was associated with predictors of local recurrence like larger tumour size, high-nuclear grade, comedo type DCIS, negative-hormone receptor status and high-Ki67 proliferation index, which was consistent with other studies. ${ }^{12,47-51}$ Local recurrence (invasive or DCIS) was associated with higher-grade, comedo necrosis and margin status. DCIS local recurrence was associated with HER2 positivity and invasive local 
The clinical and biological significance of HER2 over-expression in... IM Miligy et al.

Table 3. Association between final HER2 status and the clinicopathological parameters of pure DCIS cohort

\begin{tabular}{lllll}
\hline Parameter & \multicolumn{3}{l}{ HER2 status } & \\
& Total & Positive & Negative & $x^{2}$ ( $p$ value) \\
& $(n=646)$ & $(n=128)$ & $(n=518)$ & \\
& $n(\%)$ & $n(\%)$ & $n(\%)$ & \\
\hline
\end{tabular}

Age (years)*

Less than $40 \quad 22(3.4) \quad 7(6)$

Between $40 \quad 376(58.2) \quad 74(58) \quad 302(58) \quad(0.347)$

and 60

More than $60248(38.4) \quad 47(36) \quad 201$ (39)

Presentation

Screening $336(52) \quad 69(54) \quad 267(51) \quad 0.23$

Symptomatic $310(48) \quad 59(46) \quad 251(49) \quad$ (0.632)

DCIS size $(\mathrm{mm})$

Less than $16223(34.8) \quad 27(22) \quad 196(38) \quad 20.63$

Between $16 \quad 247(38.5) \quad 47(37) \quad 200(39) \quad(<0.0001)$

and 40

More than $40 \quad 171(26.7) \quad 52(41) \quad 119(23)$

Nuclear grade

$\begin{array}{lcccl}\text { Low } & 87(13.5) & 0(0) & 87(17) & 58.58 \\ \text { Intermediate } & 166(25.7) & 13(10) & 153(29) & (<0.0001)\end{array}$

High $\quad 393(60.8) \quad 115(90) \quad 278(54)$

Comedo necrosis

$\begin{array}{llrll}\text { Yes } & 411(63.8) & 111(87) & 300(58) & 36.28 \\ \text { No } & 233(36.2) & 17(13) & 216(42) & (<0.0001)\end{array}$

Management

$\begin{array}{lllll}\text { Mastectomy } & 339(52.5) & 84(66) & 255(49) & 11.06 \\ \text { Breast } & 307(47.5) & 44(34) & 263(51) & (\mathbf{0 . 0 0 1 )}\end{array}$

conserving

surgery

Radiotherapy

$\begin{array}{lrrrl}\text { Yes } & 95(14.7) & 19(15) & 76(15) & 0.002 \\ \text { No } & 551(85.3) & 109(85) & 442(85) & (0.961)\end{array}$

Oestrogen receptor status

$\begin{array}{lrrrl}\text { Positive } & 426(75.3) & 45(40) & 381(84) & 88.86 \\ \text { Negative } & 140(24.7) & 66(60) & 74(16) & (<0.0001)\end{array}$

Progesterone receptor status

$\begin{array}{lrrrl}\text { Positive } & 302(46.7) & 18(14) & 284(55) & 68.51 \\ \text { Negative } & 344(53.3) & 110(86) & 234(45) & (<0.0001)\end{array}$

Ki67 index

$\begin{array}{lcccc}\begin{array}{l}\text { High } \\ \text { proliferative }\end{array} & 108(23.0) & 64(59) & 97(27) & 37.46 \\ \begin{array}{l}\text { Low } \\ \text { proliferative }\end{array} & 361(77.0) & 45(41) & 263(73) & (<0.0001)\end{array}$

$p$ value in bold: significant

DCIS Ductal carcinoma in situ, $n$ number

*Age: categorised according to the Van Nuys Prognostic Index (VNPI)

recurrence was associated with tumour size. Similar to some studies, HER2 positive tumours were associated with DCIS local recurrence. $^{52,53}$

DCIS patients with HER2 amplification had higher nuclear grade lesions and therefore are probably at risk of relapse more frequently than the HER2 unamplified group, but the prognostic role of HER2 over-expression in DCIS is still not fully clarified. Some studies suggest that patients with HER2 amplified DCIS are more frequently high-nuclear grade and this aspect is related to an
Table 4. Univariate and multivariate analysis

(A) Univariate analysis for predictors of local recurrence in pure DCIS patients treated with breast conserving surgery

\begin{tabular}{|c|c|c|}
\hline Outcome & $\mathrm{HR}(95 \% \mathrm{Cl})$ & $p$ Value ${ }^{*}$ \\
\hline \multicolumn{3}{|l|}{ All local recurrence } \\
\hline Tumour size & $1.31(0.36-2.55)$ & 0.098 \\
\hline High tumour grade & $3.43(1.18-9.96)$ & 0.023 \\
\hline Radiotherapy & $0.95(0.47-1.91)$ & 0.890 \\
\hline ER status & $1.09(0.58-2.05)$ & 0.777 \\
\hline HER2 status & $0.89(0.45-1.74)$ & 0.734 \\
\hline Ki 67 status & $2.61(0.33-5.01)$ & 0.089 \\
\hline Comedo necrosis & $3.63(1.44-7.82)$ & 0.013 \\
\hline Margin width & $0.63(0.34-0.91)$ & 0.029 \\
\hline \multicolumn{3}{|l|}{ DCIS local recurrence } \\
\hline Tumour size & $1.01(0.46-2.23)$ & 0.423 \\
\hline High tumour grade & $1.90(0.21-4.72)$ & 0.569 \\
\hline Comedo necrosis & $1.24(0.73-1.99)$ & 0.372 \\
\hline Margin width & $1.83(0.15-11.96)$ & 0.180 \\
\hline Radiotherapy & $0.75(0.35-1.59)$ & 0.459 \\
\hline ER status & $0.76(0.29-1.92)$ & 0.551 \\
\hline HER2 status & $2.51(2.11-11.45)$ & 0.030 \\
\hline Ki 67 status & $2.44(0.13-2.29)$ & 0.067 \\
\hline \multicolumn{3}{|l|}{ Invasive local recurrence } \\
\hline Tumour size & $2.63(1.13-6.09)$ & 0.024 \\
\hline High tumour grade & $1.15(0.25-5.24)$ & 0.871 \\
\hline Comedo necrosis & $1.49(0.814-2.76)$ & 0.194 \\
\hline Margin width & $0.69(0.43-2.47)$ & 0.578 \\
\hline Radiotherapy & $1.17(0.35-3.86)$ & 0.780 \\
\hline ER status & $1.01(0.45-2.65)$ & 0.833 \\
\hline HER2 status & $0.49(0.17-1.41)$ & 0.184 \\
\hline Ki 67 status & $1.28(0.41-3.88)$ & 0.668 \\
\hline
\end{tabular}

(B) Multivariate Cox proportional hazards for factors associated with local recurrence in pure ductal carcinoma in situ patients

\begin{tabular}{lll}
\hline Variable & HR $(95 \% \mathrm{Cl})$ & $p$ Value \\
\hline Ductal carcinoma in situ size & $0.62(0.31-3.47)$ & 0.311 \\
Ductal carcinoma in situ grade & $2.21(1.15-70.85)$ & $\mathbf{0 . 0 3 1}$ \\
Comedo necrosis & $0.32(0.12-0.87)$ & $\mathbf{0 . 0 2 6}$ \\
Margin width & $0.89(0.11-7.46)$ & 0.911 \\
HER2+/Ki67-high group & $1.52(1.64-60.74)$ & $\mathbf{0 . 0 0 1}$ \\
\hline$D C I S$ ductal carcinoma in situ, HR hazard ratio, Cl confidence interval, ER \\
oestrogen receptor \\
* $p$ Values are corrected according to Bonferroni multiple testing correction; \\
bold facing is significant
\end{tabular}

increased risk of relapse. ${ }^{54,55}$ In fact, some studies suggest that HER2 plays a major role in the transition from DCIS to IDC, ${ }^{18,56}$ while others do not support this. ${ }^{9,57}$ In the current study, HER2 amplification was more frequent in the pure DCIS cohort $(20 \%)$ than in the mixed DCIS (15\%) despite the more prevalent highergrade DCIS in the mixed cases. HER2 positivity alone was not predictive of recurrence as a whole or as invasive disease but showed a trend to DCIS recurrence. This result is consistent with our observation that HER2 positive DCIS tend to extend within the ductal system and even in the epidermis (Paget's disease) than associated with invasive disease. It is our personal observation that when HER2-positive DCIS is associated with early invasion, that 
invasive disease may show aggressive features. Currently, HER2positive invasive tumours are treated more aggressively regardless of its size. ${ }^{58}$ This may suggest that HER2-positive DCIS tumour cells although highly proliferative are not highly invasive, whilst those that acquire invasive properties became aggressive and can metastasise.

In order to improve the risk stratification and therefore treatment recommendations, there is a need to identify potential predictors of invasive and/or non-invasive recurrence following conserving breast surgery. HER2/Ki67 positivity was a predictor of recurrence, independent of other studied clinicopathological parameters such as nuclear grade and presence of comedo necrosis. In contrast to a previous study, ${ }^{59}$ the combination of ER status with HER2 and Ki67 further added additional predictive information. This difference may be due to the differences in patient populations, or to differences in the coding of HER2 positivity. The previous study coded DCIS lesions with equivocal immunostaining for HER2 (score of $2+$ ) as positive. We performed in situ hybridisation on all equivocal cases and only lesions with amplification were coded as HER2 positive.

In the present study, HER2 expression in the invasive component was seen less frequently as compared to the DCIS component adjacent to invasive carcinoma and pure DCIS although this was of borderline statistically significant. Similarly, other studies ${ }^{54,60}$ have observed that HER2 positivity is often in patients with pure DCIS compared to those with microinvasive or invasive carcinoma. As it has been reported that patients with HER2 + invasive cancer have a poorer prognosis than those whose tumours do not express HER2, ${ }^{61}$ the higher incidence of expression found in DCIS may therefore seem paradoxical. It was postulated that HER2 expression happens during the process of atypical hyperplasia in DCIS, and loss of HER2 expression occurs as DCIS develops into invasive disease. Another hypothesis is that HER2 over-expression in invasive carcinoma does not develop from DCIS but from the associated atypical hyperplasia. ${ }^{62,63}$ Another theory explaining this phenomenon is that most invasive cancers develop from DCIS tumours, which have low expression of HER2 but are highly proliferative. ${ }^{54}$ Because such tumours progress rapidly, they are in the DCIS stage for only a short period and would therefore be under-represented in population samples. $^{54}$

In summary, $\mathrm{CISH}$ is a promising, practical test that can be used in conjunction with IHC to determine HER2 status in DCIS. IHC is easy to perform, relatively inexpensive, and able to detect a majority of breast cancer patients whose tumours have negative $(0$ or $1+)$ or positive $(3+)$ HER2 status. This $\mathrm{IHC} / \mathrm{ClSH}$ test stratification not only identifies the IHC borderline cases, but also keeps testing costs for HER2 status at a reasonable minimum. As HER2 status is not currently routinely measured in clinical practice, we aimed to show statistically significant correlations between the development of local recurrence as well as poor prognostic pathologic factors and HER2 positive DCIS which could alert clinicians. Our results might suggest considering routine assessment of HER2 in DCIS, similar to invasive breast cancer.

We also confirmed the suitability of the TMA technique for assessment of HER2 gene amplification status in tumours arising from different patients, while maintaining a representative tissue sample in each case, with good reproducibility and credible results.

To conclude, the frequency of HER2 positivity, driven by gene amplification, in DCIS is comparable to IBC and in combination with Ki67, is an independent predictor of recurrence. Our results suggest the consideration of routine assessment of HER2 status for DCIS, as it is commonly done for IBC.

Limitations of the study

This study has been conducted on TMA sections, which might underestimate the role of tumour heterogeneity. However, all cases in our cohort were carefully reviewed before TMA construction and used multiple cores for cases with heterogeneous morphology. The lower number of radiotherapy-treated cases after BCS was due to the prevalence of mastectomy in the earlier period of the study; which constituted the majority of cases.

\section{ACKNOWLEDGEMENTS}

We thank the Nottingham Health Science Biobank and Breast Cancer Now Tissue Bank for the provision of tissue samples. This research was supported and funded by the Egyptian Ministry of Higher Education and Scientific Research.

\section{AUTHOR CONTRIBUTIONS}

Conception and design: Islam M. Miligy, Emad A. Rakha and Andrew R. Green. Cases collection and IHC staining and scoring: Islam M. Miligy and Michael S. Toss. Collection and assembly of data: Islam M. Miligy, Michael S. Toss and Kylie L. Gorringe. Data analysis and interpretation: Islam M. Miligy, Kylie L. Gorringe, Michael S. Toss, Andrew H. S. Lee, Ian O. Ellis, Andrew R. Green and Emad A. Rakha Manuscript writing: Islam M. Miligy, Michael S. Toss, Kylie L. Gorringe, Andrew H. S. Lee, Ian O. Ellis, Andrew R. Green and Emad A. Rakha All authors contributed to writing the paper manuscript and approved the final version.

\section{ADDITIONAL INFORMATION}

Supplementary information is available for this paper at https://doi.org/10.1038/ s41416-019-0436-3.

Competing interests: The authors declare that they have no competing interests.

Ethical approval: This work obtained ethics approval by the North West - Greater Manchester Central Research Ethics Committee under the title; Nottingham Health Science Biobank (NHSB), reference number 15/NW/068

Data availability: The authors confirm the data that has been used in this work is available on reasonable request.

Note: This work is published under the standard license to publish agreement. After 12 months the work will become freely available and the license terms will switch to a Creative Commons Attribution 4.0 International (CC BY 4.0)

Publisher's note: Springer Nature remains neutral with regard to jurisdictional claims in published maps and institutional affiliations.

\section{REFERENCES}

1. Lari, S. A. \& Kuerer, H. M. Biological markers in DCIS and risk of breast recurrence: a systematic review. J. Cancer 2, 232-261 (2011).

2. Narod SA, lqbal J, Giannakeas V, Sopik V, Sun P. Breast cancer mortality after a diagnosis of ductal carcinoma in situ. JAMA Oncol. 1, 888-896 (2015).

3. Rakovitch, E., Nofech-Mozes, S., Hanna, W., Baehner, F. L., Saskin, R., Butler, S. M. et al. A population-based validation study of the DCIS Score predicting recurrence risk in individuals treated by breast-conserving surgery alone. Breast Cancer Res. Treat. 152, 389-398 (2015).

4. Bartlett, J. M., Nofech-Moses, S. \& Rakovitch, E. Ductal carcinoma in situ of the breast: can biomarkers improve current management? Clin. Chem. 60, 60-67 (2014).

5. Fleischer, T., Frigessi, A., Johnson, K. C., Edvardsen, H., Touleimat, N., Klajic, J. et al. Genome-wide DNA methylation profiles in progression to in situ and invasive carcinoma of the breast with impact on gene transcription and prognosis. Genome Biol. 15, 435 (2014).

6. Burstein, H. J., Polyak, K., Wong, J. S., Lester, S. C. \& Kaelin, C. M. Ductal carcinoma in situ of the breast. N. Engl. J. Med. 350, 1430-1441 (2004).

7. Zhang $X$, Dai H, Liu B, Song F, Chen K. Predictors for local invasive recurrence of ductal carcinoma in situ of the breast: a meta-analysis. Eur. J. Cancer Prev. 25, 19-28 (2015).

8. Silverstein, M. J. Current controversies in ductal carcinoma in situ of the breast: summary from the Lynn Sage Breast Cancer Symposium. J. Am. Coll. Surg. 197, 115-118 (2003a).

9. Borgquist, S., Zhou, W., Jirstrom, K., Amini, R. M., Sollie, T., Sorlie, T. et al. The prognostic role of HER2 expression in ductal breast carcinoma in situ (DCIS); a population-based cohort study. BMC Cancer 15, 468 (2015). 
10. Silverstein, M. J. The University of Southern California/Van Nuys prognostic index for ductal carcinoma in situ of the breast. Am. J. Surg. 186, 337-343 (2003b).

11. Solin, L. J., Gray, R., Baehner, F. L., Butler, S. M., Hughes, L. L., Yoshizawa, C. et al. A multigene expression assay to predict local recurrence risk for ductal carcinoma in situ of the breast. J. Natl Cancer Inst. 105, 701-710 (2013).

12. Latta, E. K., Tjan, S., Parkes, R. K. \& O'Malley, F. P. The role of HER2/neu overexpression/amplification in the progression of ductal carcinoma in situ to invasive carcinoma of the breast. Mod. Pathol. 15, 1318-1325 (2002).

13. Allred, D. C., Clark, G. M., Tandon, A. K., Molina, R., Tormey, D. C., Osborne, C. K. et al. HER-2/neu in node-negative breast cancer: prognostic significance of overexpression influenced by the presence of in situ carcinoma. J. Clin. Oncol. 10, 599-605 (1992)

14. Horimoto, Y., Tokuda, E., Arakawa, A., Kosaka, T., Saito, M. \& Kasumi, F. Significance of HER2 protein examination in ductal carcinoma in situ. J. Surg. Res. 167, e205-e210 (2011).

15. Roses, R. E., Paulson, E. C., Sharma, A., Schueller, J. E., Nisenbaum, H., Weinstein, S. et al. HER-2/neu overexpression as a predictor for the transition from in situ to invasive breast cancer. Cancer Epidemiol. Biomark. Prev. 18, 1386-1389 (2009).

16. Allred, D. C., Anderson, S. J., Paik, S., Wickerham, D. L., Nagtegaal, I. D., Swain, S. M. et al. Adjuvant tamoxifen reduces subsequent breast cancer in women with estrogen receptor-positive ductal carcinoma in situ: a study based on NSABP protocol B-24. J. Clin. Oncol. 30, 1268-1273 (2012).

17. Tamimi, R. M., Baer, H. J., Marotti, J., Galan, M., Galaburda, L., Fu, Y. et al. Comparison of molecular phenotypes of ductal carcinoma in situ and invasive breast cancer. Breast Cancer Res. 10, R67 (2008).

18. Williams, K. E., Barnes, N. L., Cramer, A., Johnson, R., Cheema, K., Morris, J. et al. Molecular phenotypes of DCIS predict overall and invasive recurrence. Ann. Oncol. 26, 1019-1025 (2015).

19. Hanna, W., Kahn, H. J. \& Trudeau, M. Evaluation of HER-2/neu (erbB-2) status in breast cancer: from bench to bedside. Mod. Pathol. 12, 827-834 (1999).

20. Jimenez, R. E., Wallis, T., Tabasczka, P. \& Visscher, D. W. Determination of Her-2/ Neu status in breast carcinoma: comparative analysis of immunohistochemistry and fluorescent in situ hybridization. Mod. Pathol. 13, 37-45 (2000).

21. Perez, E. A., Cortes, J., Gonzalez-Angulo, A. M. \& Bartlett, J. M. HER2 testing current status and future directions. Cancer Treat. Rev. 40, 276-284 (2014).

22. Pfitzner, B. M., Lederer, B., Lindner, J., Solbach, C., Engels, K., Rezai, M. et al. Clinical relevance and concordance of HER2 status in local and central testing-an analysis of 1581 HER2-positive breast carcinomas over 12 years. Mod. Pathol. 31, 607-615 (2018).

23. Pothos, A., Plastira, K., Plastiras, A., Vlachodimitropoulos, D., Goutas, N. \& Angelopoulou, R. Comparison of chromogenic in situ hybridisation with fluorescence in situ hybridisation and immunohistochemistry for the assessment of her- $2 /$ neu oncogene in archival material of breast carcinoma. Acta Histochem. Cytochem. 41, 59-64 (2008).

24. Penault-Llorca, F., Bilous, M., Dowsett, M., Hanna, W., Osamura, R. Y., Ruschoff, J. et al. Emerging technologies for assessing HER2 amplification. Am. J. Clin. Pathol. 132, 539-548 (2009).

25. Lester, S. C., Bose, S., Chen, Y. Y., Connolly, J. L., de Baca, M. E., Fitzgibbons, P. L. et al. Protocol for the examination of specimens from patients with ductal carcinoma in situ of the breast. Arch. Pathol. Lab. Med. 133, 15-25 (2009).

26. Miligy, I. M., Gorringe, K. L., Toss, M. S., Al-Kawaz, A. A., Simpson, P., Diez-Rodriguez, M., et al. Thioredoxin-interacting protein is an independent risk stratifier for breast ductal carcinoma in situ. Mod. Pathol. 12, 1807-1815 (2018).

27. Rakha, E. A., Pinder, S. E., Bartlett, J. M., Ibrahim, M., Starczynski, J., Carder, P. J. et al. Updated UK recommendations for HER2 assessment in breast cancer. J. Clin. Pathol. 68, 93-99 (2015).

28. Wolff, A. C., Hammond, M. E., Hicks, D. G., Dowsett, M., McShane, L. M., Allison, K. $H$. et al. Recommendations for human epidermal growth factor receptor 2 testing in breast cancer: American Society of Clinical Oncology/College of American Pathologists clinical practice guideline update. J. Clin. Oncol. 31, 3997-4013 (2013).

29. Hammond, M. E., Hayes, D. F., Wolff, A. C., Mangu, P. B. \& Temin, S. American Society of Clinical Oncology/College of American Pathologists Guideline recommendations for immunohistochemical testing of estrogen and progesterone receptors in breast cancer. J. Oncol. Pract. 6, 195-197 (2010a).

30. Goldhirsch, A., Wood, W. C., Coates, A. S., Gelber, R. D., Thurlimann, B. \& Senn, H. J. Strategies for subtypes--dealing with the diversity of breast cancer: highlights of the St. Gallen International Expert Consensus on the Primary Therapy of Early Breast Cancer 2011. Ann. Oncol. 22, 1736-1747 (2011).

31. Madrid, M. A. \& Lo, R. W. Chromogenic in situ hybridization (CISH): a novel alternative in screening archival breast cancer tissue samples for HER-2/neu status. Breast Cancer Res. 6, R593-R600 (2004)

32. Kakar, S., Puangsuvan, N., Stevens, J. M., Serenas, R., Mangan, G., Sahai, S. et al. HER-2/neu assessment in breast cancer by immunohistochemistry and fluorescence in situ hybridization: comparison of results and correlation with survival. Mol. Diagn. 5, 199-207 (2000).

33. Thomson, T. A., Hayes, M. M., Spinelli, J. J., Hilland, E., Sawrenko, C., Phillips, D. et al. HER-2/neu in breast cancer: interobserver variability and performance of immunohistochemistry with 4 antibodies compared with fluorescent in situ hybridization. Mod. Pathol. 14, 1079-1086 (2001).

34. Malicka-Durczak, A., Korski, K. \& Ibbs, M. Comparison of in situ hybridization methods for the assessment of HER-2/neu gene amplification status in breast cancer using a tissue microarray. Rep. Pract. Oncol. Radiother. 17, 44-49 (2012).

35. Tabarestani, S., Ghaderian, S. M. \& Rezvani, H. Detection of gene amplification by multiplex ligation-dependent probe amplification in comparison with in situ hybridization and immunohistochemistry. Asian Pac. J. Cancer Prev. 16, 7997-8002 (2015).

36. Gong, Y., Sweet, W., Duh, Y. J., Greenfield, L., Fang, Y., Zhao, J. et al. Chromogenic in situ hybridization is a reliable method for detecting HER2 gene status in breast cancer: a multicenter study using conventional scoring criteria and the new ASCO/CAP recommendations. Am. J. Clin. Pathol. 131, 490-497 (2009).

37. Garcia-Caballero, T., Grabau, D., Green, A. R., Gregory, J., Schad, A., Kohlwes, E. et al. Determination of HER2 amplification in primary breast cancer using dualcolour chromogenic in situ hybridization is comparable to fluorescence in situ hybridization: a European multicentre study involving 168 specimens. Histopathology 56, 472-480 (2010).

38. Gruver, A. M., Peerwani, Z. \& Tubbs, R. R. Out of the darkness and into the light: bright field in situ hybridisation for delineation of ERBB2 (HER2) status in breast carcinoma. J. Clin. Pathol. 63, 210-219 (2010).

39. Arnould, L., Denoux, Y., MacGrogan, G., Penault-Llorca, F., Fiche, M., Treilleux, I. et al. Agreement between chromogenic in situ hybridisation (CISH) and FISH in the determination of HER2 status in breast cancer. Br. J. Cancer 88, 1587-1591 (2003).

40. Zhao, J., Wu, R., Au, A., Marquez, A., Yu, Y. \& Shi, Z. Determination of HER2 gene amplification by chromogenic in situ hybridization (CISH) in archival breast carcinoma. Mod. Pathol. 15, 657-665 (2002).

41. Tubbs, R. R., Pettay, J. D., Roche, P. C., Stoler, M. H., Jenkins, R. B. \& Grogan, T. M. Discrepancies in clinical laboratory testing of eligibility for trastuzumab therapy: apparent immunohistochemical false-positives do not get the message. J. Clin. Oncol. 19, 2714-2721 (2001).

42. Bartlett, J. M., Campbell, F. M. \& Mallon, E. A. Determination of HER2 amplification by in situ hybridization: when should chromosome 17 also be determined? Am. J. Clin. Pathol. 130, 920-926 (2008).

43. Sapino, A., Coccorullo, Z., Cassoni, P., Ghisolfi, G., Gugliotta, P., Bongiovanni, M et al. Which breast carcinomas need HER-2/neu gene study after immunohistochemical analysis? Results of combined use of antibodies against different cerbB2 protein domains. Histopathology 43, 354-362 (2003).

44. Jourdan, F., Sebbagh, N., Comperat, E., Mourra, N., Flahault, A., Olschwang, S. et al. Tissue microarray technology: validation in colorectal carcinoma and analysis ofp53, hMLH1, and hMSH2 immunohistochemical expression. Virchows Arch. 443, 115-121 (2003).

45. Mulrane, L., Gallagher, W. M. \& O'Connor, D. P. Assessment of significance of novel proteins in breast cancer using tissue microarray technology. Methods Mol. Biol. 1501, 311-325 (2017).

46. Camp, R. L., Charette, L. A. \& Rimm, D. L. Validation of tissue microarray technology in breast carcinoma. Lab. Invest. 80, 1943-1949 (2000).

47. DiGiovanna, M. P., Chu, P., Davison, T. L., Howe, C. L., Carter, D., Claus, E. B. et al. Active signaling by HER-2/neu in a subpopulation of HER-2/neu-overexpressing ductal carcinoma in situ: clinicopathological correlates. Cancer Res. 62, 6667-6673 (2002).

48. Lebeau, A., Unholzer, A., Amann, G., Kronawitter, M., Bauerfeind, I., Sendelhofert, A. et al. EGFR, HER-2/neu, cyclin D1, p21 and p53 in correlation to cell proliferation and steroid hormone receptor status in ductal carcinoma in situ of the breast. Breast Cancer Res. Treat. 79, 187-198 (2003).

49. Claus, E. B., Chu, P., Howe, C. L., Davison, T. L., Stern, D. F., Carter, D. et al Pathobiologic findings in DCIS of the breast: morphologic features, angiogenesis, HER-2/neu and hormone receptors. Exp. Mol. Pathol. 70, 303-316 (2001).

50. Kuerer, H. M., Buzdar, A. U., Mittendorf, E. A., Esteva, F. J., Lucci, A., Vence, L. M. et al. Biologic and immunologic effects of preoperative trastuzumab for ductal carcinoma in situ of the breast. Cancer 117, 39-47 (2011).

51. Holmes, P., Lloyd, J., Chervoneva, I., Pequinot, E., Cornfield, D. B., Schwartz, G. F. et al. Prognostic markers and long-term outcomes in ductal carcinoma in situ of the breast treated with excision alone. Cancer 117, 3650-3657 (2011).

52. Rakovitch, E., Nofech-Mozes, S., Hanna, W., Narod, S., Thiruchelvam, D., Saskin, R et al. HER2/neu and Ki-67 expression predict non-invasive recurrence following breast-conserving therapy for ductal carcinoma in situ. Br. J. Cancer 106, 1160-1165 (2012). 
53. Han, K., Nofech-Mozes, S., Narod, S., Hanna, W., Vesprini, D., Saskin, R. et al. Expression of HER2neu in ductal carcinoma in situ is associated with local recurrence. Clin. Oncol. 24, 183-189 (2012).

54. Rohilla, M., Bal, A., Singh, G. \& Joshi, K. Prediction of heterogeneity in breast cancer immunophenotype at ductal carcinoma in situ stage? J. Cancer Res. Ther. 12, 1249-1256 (2016).

55. Millis, R. R., Bobrow, L. G. \& Barnes, D. M. Immunohistochemical evaluation of biological markers in mammary carcinoma in situ: correlation with morphological features and recently proposed schemes for histological classification. Breast 14, 16 (1996).

56. Burkhardt, L., Grob, T. J., Hermann, I., Burandt, E., Choschzick, M., Janicke, F. et al. Gene amplification in ductal carcinoma in situ of the breast. Breast Cancer Res. Treat. 123, 757-765 (2010).

57. Kim, J. Y., Park, K., Kang, G., Kim, H. J., Gwak, G. \& Shin, Y. J. Predictors of recurrent ductal carcinoma in situ after breast-conserving surgery. J. Breast Cancer 19, 185-190 (2016).

58. Joerger, M., Thurlimann, B. \& Huober, J. Small HER2-positive, node-negative breast cancer: who should receive systemic adjuvant treatment? Ann. Oncol. 22, 17-23 (2011).
59. Kerlikowske, K., Molinaro, A. M., Gauthier, M. L., Berman, H. K., Waldman, F., Bennington, J. et al. Biomarker expression and risk of subsequent tumors after initial ductal carcinoma in situ diagnosis. J. Natl Cancer Inst. 102, 627-637 (2010).

60. Park, K., Han, S., Kim, H. J., Kim, J. \& Shin, E. HER2 status in pure ductal carcinoma in situ and in the intraductal and invasive components of invasive ductal carcinoma determined by fluorescence in situ hybridization and immunohistochemistry. Histopathology 48, 702-707 (2006).

61. Rom, J., Schumacher, C., Gluz, O., Hofler, J., Eidt, S., Domschke, C. et al. Association of HER2 overexpression and prognosis in small (T1N0) primary breast cancers. Breast Care 8, (208-214 (2013).

62. Hoque, A., Sneige, N., Sahin, A. A., Menter, D. G., Bacus, J. W., Hortobagyi, G. N. et al. Her-2/neu gene amplification in ductal carcinoma in situ of the breast. Cancer Epidemiol. Biomark. Prev. 11, 587-590 (2002).

63. Mylonas, I., Makovitzky, J., Jeschke, U., Briese, V., Friese, K. \& Gerber, B. Expression of Her2/neu, steroid receptors (ER and PR), Ki67 and p53 in invasive mammary ductal carcinoma associated with ductal carcinoma in situ (DCIS) versus invasive breast cancer alone. Anticancer Res. 25(3a), 1719-1723 (2005). 